\title{
Prevalence and Factors Associated with Dysmenorrhea in Parakou, Benin
}

\author{
Mahublo Vinadou Vodouhe1, Rachidi Sidi Imorou1, Raoul Atade1, Kabibou Salifou', \\ Ulrich Vignonzan1, Nouessewa Fanny Maryline Hounkponou1, Koffi N'tcha², \\ Awadé Afoukou Achille Obossou ${ }^{1}$
}

${ }^{1}$ UFR of Gynecology and Obstetrics, Faculty of Medicine, University of Parakou, Parakou, Benin

${ }^{2}$ Laboratory for Studies and Research on Social Dynamics and Local Development, Parakou, Benin

Email: hublo.vinadou@gmail.com

How to cite this paper: Vodouhe, M.V., Sidi Imorou, R., Atade, R., Salifou, K., Vignonzan, U., Hounkponou, N.F.M., N’tcha, K. and Obossou, A.A.A. (2020) Prevalence and Factors Associated with Dysmenorrhea in Parakou, Benin. Open Journal of Obstetrics and Gynecology, 10, 1000-1010. https://doi.org/10.4236/ojog.2020.1080095

Received: June 11, 2020

Accepted: August 3, 2020

Published: August 6, 2020

Copyright $\odot 2020$ by author(s) and Scientific Research Publishing Inc. This work is licensed under the Creative Commons Attribution International License (CC BY 4.0).

http://creativecommons.org/licenses/by/4.0/

\begin{abstract}
Background: Dysmenorrhea is a common symptom of gynecology that causes social and economic disruption in the lives of women who suffer from it. But many of them consider it inevitable and do not consult a health worker. Knowing the factors associated with this condition could help reduce its frequency and impact. Objective: To determine the prevalence and factors associated with dysmenorrhea in Benin in 2017. Method: This was a cross-sectional, descriptive and analytical study from 17 April to 17 May 2017. It involved 822 women aged 15 to 44 selected by a cluster sampling technique. Information on socio-demographic characteristics, functional signs, and medical care was collected using questionnaires. The relationship between these factors and dysmenorrhea was analyzed using the chi 2 test. The protocol was accepted by the ethics committee for biomedical research of the University of Parakou. The patients had given their informed consent. Results: The prevalence of dysmenorrhea was $72.6 \%(95 \% \mathrm{CI}=[69.48 ; 75.57])$. Dysmenorrhea was predominantly primary (75\%). 6.9\% of women suffering from dysmenorrhea were using oral contraceptive. Asthenia and irritability are the most common accompanying signs of dysmenorrhea in $68.2 \%$ and $54.4 \%$ of cases respectively. Age, marital status, socio-professional occupation, educational level and parity were the factors associated $(P<0.001)$ with dysmenorrhea. A proportion of $87.3 \%$ of women suffering from dysmenorrhea had no medical care. Conclusion: The prevalence of dysmenorrhea is high in our climes. It would be important to raise awareness among women for medical and psychosocial care taking into account associated factors.
\end{abstract}

\section{Keywords}

Dysmenorrhea, Menstruation, Associated Factors 


\section{Introduction}

Dysmenorrhea is all the pelvic pains preceding or accompanying menstruation [1]. Classically, a distinction is made between primary dysmenorrhea and secondary dysmenorrhea. Primary dysmenorrhoea begins in adolescence, in the months or years following menarches and is almost always without an organic cause. Secondary dysmenorrhoea appears at a late stage of puberty, most often in women over 30 years of age, and often has an organic cause. It is a real public health issue. The prevalence of dysmenorrhea varies from $43 \%$ to $90 \%$ worldwide depending on the study population and the study method [2] [3]. According to the results of a study carried out in France, its prevalence was $66 \%$ of young women and 55\% of adolescent girls [4]. It is accompanied by an altered quality of life and is a frequent cause of illness and absenteeism from work among women [5] [6]. The literature is diverse on the factors associated with dysmenorrhea [5] [6]. Today effective treatments exist. However, many women do not treat themselves believing that it is inevitable [1]. This study aimed at determining the prevalence and the factors associated with dysmenorrhea among women of childbearing age in the city of Parakou, Benin in 2017.

\section{Study Methods}

\section{Type of study}

It was a cross-sectional, descriptive and analytical study conducted over a 1-month period: from April 17 to May 17, 2017.

\section{Target population and sampling procedure}

The target population consisted of women aged 15 to 44, living in the neighbourhoods of the three districts of the municipality of Parakou. The following were excluded from our study: women who have had amenorrhea for at least one year, or who had been living in the town of Parakou for less than 6 months.

In view of the $78.35 \%$ prevalence of dysmenorrhea [7] in a survey carried out in a population of young adolescent girls in Parakou, and an accuracy of 3\%, the size of the sample is calculated according to Schwartz's formula.

$$
n=\frac{Z \alpha^{2} * p *(1-p)}{i^{2}}
$$

with $\alpha=0.05: p$ value of $Z \alpha=1.96 ; p=78.35 \% ; i=3 \%$ : the desired accuracy; n: the expected sample size $n=724.05$ women. Taking into account the rate of non-respondents, a $10 \%$ margin was calculated and added to the initial size. Thus the minimum sample size within the framework of the survey was 796 women.

The sampling technique was the cluster survey. The basis of this survey was composed of the list of the 41 neighbourhoods in the municipality of Parakou with their respective target populations. We selected 29 clusters. The size of each cluster was then $26.53(796 / 30)$, roughly equal to 27 individuals per cluster, which redefined the size of the sample to $810(30 \times 27)$. To select the thirty $(30)$ clusters, a cumulative staffing table of the target population of each neighbour- 
hood/village was drawn up. Then, the cluster pitch $k=8516$ calculated, was obtained by making the ratio between the size of the cumulative target population and the total number of clusters $(255,478 / 30)$. The onset (onset $=2597)$ was obtained by randomly drawing a number between 1 and the cluster pitch $(k)$ using the Epitable module of the Epi6 software French version 6.04. The number drawn enabled us to locate the first cluster (Banikanni area). The second cluster was obtained by adding to the onset, the cluster pitch $(2597+8516=11,113)$. To each new result, the cluster pitch $(k)$ was added until the 30 clusters were obtained. Within the city neighbourhood where a cluster was drawn, the interviewer would stand in the centre of the district and draw a direction by the turned pen method. Thus, in that direction, out of two compounds, only one was drawn by lot beginning with the one on the right side. Within each compound selected, numbers were assigned to each household on pieces of paper and only one was drawn randomly. In each household selected, a woman was drawn from the list of eligible women present in the household as the collection team passed by. If the expected number of subjects planned was not reached in this direction, the interviewer returned to the center of the neighbourhood and walked in the opposite direction to the first one drawing the sample in the same way.

\section{Variables}

The dependent variable of the survey was dysmenorrhea in women aged 15 to 44. It is a binary qualitative variable with yes/no modalities. The independent variables were: the type of dysmenorrhea, The women's socio-demographic characteristics (age, marital status, educational level, socio-professional occupation, parity, body mass index (BMI)), the type of contraceptive method used, the signs associated with dysmenorrhea, the number of days of absence from work caused by dysmenorrhea, the therapeutic attitude of women (place of request for care, type of treatment). The information on these different variables was obtained after a review of the literature [5] [6].

\section{- Operational definitions}

- Dysmenorrhea: pain in the lower abdomen or lower back occurring before and/or during menstruation.

- Primary dysmenorrhea: dysmenorrhea occurring in the year following menarche, without organic support.

- Secondary dysmenorrhea: dysmenorrhea occurring after a period when menstruation was normal.

\section{Data collection}

The data collection tool was a standardized questionnaire taking into account the above-mentioned variables. The data collection method was a structured interview that was conducted by 3 students from the midwifery school of the University of Parakou with the participating women. The data collection was conducted over a period of 1 month: from April 17 to May 17, 2017.

\section{Data analysis}

The data collected had been entered using Epi-data 3.1 software. The data 
analysis was done using Epi-data Analysis version 2.2.2.182 software. Quantitative variables were expressed in average and standard deviations and as a percentage for qualitative variables. The different frequencies were compared using the chi $^{2}$ test. A $p<0.05$ was considered to be statistically significant.

\section{Ethical considerations}

The study protocol was submitted to the Local Ethics Committee for Biomedical Research of the University of Parakou which gave its approval to carry out this study. (REF: 0063/CLERB-UP/P/SP/R/SA). The participants were informed about the objective and the protocol of the study. Their informed consent was obtained prior to data collection.

\section{Result}

At the end of the survey the minimum sample size initially set at 810 was 822 , giving a participation rate of $822 / 810(101 \%)$.

The prevalence of dysmenorrhea was 72.6\% (597/822); (CI95\% = [69.5; 75.6]). Dysmenorrhea was predominantly primary $(75 \%)$. There was $25 \%$ of secondary dysmenorrhea.

Socio-demographic characteristics of women suffering from dysmenorrhea

The average age was $22.2 \pm 6.7$ years old; with extremes ranging from 15 to 46 years old. A proportion of $64.2 \%$ of patients were pupils or students; $75.7 \%$ were single women; $58.6 \%$ had a normal weight (Table 1 ).

Use of contraceptive methods by women suffering from dysmenorrhea

A contraceptive method was used by 34.3\% (205/597) of women suffering from dysmenorrhea. For $12.4 \%$ (74/597) of them, the methods were hormonal. $6.9 \%(41 / 497)$ of women suffering from dysmenorrhea used oral contraceptive (pill) (Table 2).

\section{Accompanying signs of dysmenorrhea and absence from work}

The main accompanying signs of dysmenorrhea were asthenia (68.2\%) and nervousness (54.4\%) (Table 3). We identified 32.7\% cases of absence from work related to dysmenorrhea with an average number of days of absence from work of $2.0 \pm 1.1$ days. A proportion of $77.4 \%$ of absenteeism was between 1 - 2 days.

\section{Factors associated with dysmenorrhea}

There was a significant association between age and the occurrence of dysmenorrhea $(p<0.001)$. In fact, women under the age of 20 were 1.5 times more likely than others to develop dysmenorrhea.

A significant association was observed between parity and dysmenorrhea $(p<$ 0.001 ). The risk of dysmenorrhea was 1.8 times greater in nulliparous women.

The marital status presented a statistically significant link with dysmenorrhea $(p<0.001)$. It is found out that single women were 1.3 times more likely than others to have dysmenorrhea.

Dysmenorrhea was also significantly associated with the education level $(p<$ 0.001 ). Compared to women with no education, those with secondary education level were 1.4 times more likely to develop dysmenorrhea. 
Table 1. Distribution of women with dysmenorrhea according to their socio-demographic characteristics.

\begin{tabular}{|c|c|c|}
\hline & $\begin{array}{l}\text { Number } \\
\mathrm{N}=597\end{array}$ & Frequency (\%) \\
\hline \multicolumn{3}{|l|}{ Age } \\
\hline$<20$ years & 292 & 48.9 \\
\hline$[20-30]$ years & 235 & 39.4 \\
\hline$[30-40]$ years & 54 & 09.0 \\
\hline$[40-50]$ years & 16 & 2.7 \\
\hline \multicolumn{3}{|l|}{ Body Mass Index } \\
\hline Leanness & 111 & 18.6 \\
\hline Normal & 350 & 58.6 \\
\hline Overweight & 91 & 15.2 \\
\hline Obesity & 45 & 7.5 \\
\hline \multicolumn{3}{|l|}{ Educational level } \\
\hline With no schooling & 58 & 09.7 \\
\hline Primary level & 64 & 10.7 \\
\hline Secondary level & 431 & 72.2 \\
\hline University level & 44 & 07.4 \\
\hline \multicolumn{3}{|c|}{ Socio-professional Occupation } \\
\hline Pupil/student & 383 & 64.2 \\
\hline Civil servant & 34 & 5.7 \\
\hline Housewife & 42 & 7.0 \\
\hline Trader & 86 & 14.4 \\
\hline Craftswoman & 52 & 8.7 \\
\hline Parity & $\mathrm{n}=173$ & \\
\hline Nulliparous & 20 & 11.6 \\
\hline Primiparous & 51 & 29.5 \\
\hline Low parity (2 to 3 ) & 73 & 42.2 \\
\hline Multiparous (4 to 5) & 26 & 15.3 \\
\hline Grand multiparous $(>5)$ & 3 & 1.7 \\
\hline
\end{tabular}

Table 2. Distribution of women with dysmenorrhea according to their type of contraceptive method.

\begin{tabular}{|c|c|c|}
\hline Methods used & Number & Frequency (\%) \\
\hline \multicolumn{3}{|c|}{ Hormonal method } \\
\hline Pill & 41 & 6.9 \\
\hline Progestin injectable or implant & 33 & 5.5 \\
\hline \multicolumn{3}{|c|}{ Nonhormonal method } \\
\hline CIUD (Copper Intrauterine Device) & 15 & 2.5 \\
\hline Condom & 116 & 19.4 \\
\hline Lack of contraception & 392 & 65.7 \\
\hline
\end{tabular}


Table 3. Distribution of women with dysmenorrhea according to the accompanying signs of their dysmenorrheal.

\begin{tabular}{ccc}
\hline & Number & Frequency (\%) \\
& $\mathbf{N}=\mathbf{5 9 7}$ & 28.3 \\
\hline Nausea/vomiting & 169 & 25.6 \\
Diarrhea/constipation & 153 & 35.7 \\
Headache & 213 & 49.1 \\
Vertigo & 293 & 25.3 \\
Leg cramp & 151 & $\mathbf{6 8 . 2}$ \\
Asthenia & 407 & 44.7 \\
Breast pain & 267 & 46.4 \\
Anxiety & 277 & $\mathbf{5 4 . 4}$ \\
Nervousness & 325 & \\
\hline
\end{tabular}

Similarly, professional occupation and dysmenorrhea were significantly associated $(p<0.001)$. It was then noted that compared to housewives, civil servants and pupils/students had 1.4 and 1.3 times higher risks, respectively, of having dysmenorrhea.

However, no significant association was observed between BMI and dysmenorrhea $(p>0.05)$ (Table 4).

\section{Management of dysmenorrhea}

A proportion of $87.3 \%$ (521/597) of women suffering from dysmenorrhea had never been medically treated for their dysmenorrhea: There was a proportion of $30 \%(179 / 597)$ of therapeutic abstention; the others practiced self-medication and traditional medicine.

\section{Discussion}

Dysmenorrhea is a common health issue. Its prevalence in our study was $72.6 \%$. This result is close to those found by Unsal [7] (72.7\%) in 2009, and Gumanga [8] (74.4\%) in 2012. On the other hand, it is higher than those of Jang [9] (58.8\%) in 2013, Baghianimoghadam [10] (38.1\%) in 2012, and Tshabu Aguemon [11] (65.6\%) in 2014. The differences in prevalences may be due to the difference in the populations studied.

The average age of the women interviewed was $22.2 \pm 6.7$ years old. Women under 20 years old accounted for $48.9 \%$. Our results were similar to those of Unsal [7] who had an average age of $20.8 \pm 1.8$ years old with $45.4 \%$ of the pupils interviewed who were under 20 years old. However Tavallaee [12] and Jang [9] reported higher average ages respectively $29.5 \pm 6$ years old and $25.5 \pm 4.4$ years old. These differences could be explained by the different age limits of the populations studied.

We found out that single women had more dysmenorrhea $(p<0.001)$. The majority of women with dysmenorrhea in our sample were single $75.7 \%$. Baghianimoghadam [10] made the same observation: the proportion of single women was higher in their series (84.3\%). 
Table 4. Factors associated with dysmenorrheal.

\begin{tabular}{|c|c|c|c|c|c|}
\hline & \multirow{2}{*}{$\begin{array}{c}\text { Total } \\
\mathrm{N}=822\end{array}$} & \multicolumn{2}{|c|}{ Dysmenorrhea } & \multirow{2}{*}{ PR } & \multirow{2}{*}{ CI95\% PR } \\
\hline & & $\mathrm{n}=597$ & $\%$ & & \\
\hline Age & & & & & $P<0.001$ \\
\hline$<20$ years & 364 & 292 & 80.2 & 1.5 & {$[1.2-1.8]$} \\
\hline$[20-30]$ years & 333 & 235 & 70.6 & 1.3 & {$[1.1-1.6]$} \\
\hline$[30-40]$ years & 99 & 54 & 54.5 & 1 & Reference \\
\hline$[40-50]$ years & 26 & 16 & 61.5 & 1.1 & {$[0.8-1.6]$} \\
\hline Parity & & & & & $P<0.001$ \\
\hline Nulliparous & 569 & 444 & 78.0 & 1.8 & {$[1.3-2.4]$} \\
\hline Primiparous & 77 & 51 & 66.2 & 1.5 & {$[1.1-2.1]$} \\
\hline Low parity & 112 & 73 & 65.2 & 1.5 & {$[1.1-2.0]$} \\
\hline Multiparous & 59 & 26 & 41.1 & 1 & Reference \\
\hline Grand multiparous & 5 & 3 & 60.0 & 1.4 & {$[0.6-2.9]$} \\
\hline Marital status & & & & & $P<0.001$ \\
\hline Single & 579 & 452 & 78.1 & 1.3 & {$[1.2-1.5]$} \\
\hline Married/with a partner & 234 & 139 & 59.4 & 1 & Reference \\
\hline Divorced & 9 & 6 & 66.7 & 1.1 & {$[0.7-1.8]$} \\
\hline Educational level & & & & & $P<0.001$ \\
\hline With no schooling & 105 & 58 & 55.2 & 1 & Reference \\
\hline Primary level & 99 & 64 & 64.6 & 1.2 & {$[0.9-1.5]$} \\
\hline Secondary level & 558 & 431 & 77.2 & 1.4 & {$[1.2-1.7]$} \\
\hline University level & 60 & 44 & 73.3 & 1.3 & {$[1.0-1.7]$} \\
\hline Body Mass Index & & & & & $P=0.1366$ \\
\hline Leanness & 146 & 111 & 76.0 & 1.2 & {$[1.0-1.5]$} \\
\hline Normal & 476 & 350 & 73.5 & 1.2 & {$[1.0-1.4]$} \\
\hline Overweight & 127 & 91 & 71.7 & 1.2 & {$[0.9-1.4]$} \\
\hline Obesity & 73 & 45 & 61.6 & 1 & Reference \\
\hline Socio-professional occupation & & & & & $P<0.001$ \\
\hline Pupil/student & 495 & 383 & 77.4 & 1.3 & {$[1.1-1.6]$} \\
\hline Civil servant & 41 & 34 & 82.9 & 1.4 & {$[1.1-1.8]$} \\
\hline Housewife & 73 & 42 & 57.5 & 1 & Reference \\
\hline Trader & 130 & 86 & 66.2 & 1.2 & {$[0.9-1.4]$} \\
\hline Craftswoman & 83 & 52 & 62.7 & 1.1 & {$[0.8-1.4]$} \\
\hline
\end{tabular}

Socio-professional occupation was significantly related to dysmenorrhea $(p<$ $0.001)$ with a higher proportion of women $(82.9 \%)$ with dysmenorrhea who were civil servants. This finding could be explained by the stress to which certain women civil servants are subjected. Laszlo [13] and Nohara [14] reported a posi- 
tive association between stress and the risk of dysmenorrhea among female workers. Stress-related hormones appear to influence prostaglandin synthesis, which plays a major role in $80 \%-90 \%$ of dysmenorrhea [15] [16].

In our series there was an association between parity and dysmenorrhea $(p=$ 0.02 ). The nulliparous women had 1.8 times more risk of dysmenorrhea compared to multiparous women. This result is similar to that of a previous study which showed that parity is a protective factor against dysmenorrhea [16]. Theories like the reduction of prostaglandins after delivery, pelvic floor injury, disappearance of the uterine adrenergic nerves after pregnancy and reduction of uterine noradrenaline during the last trimester of pregnancy were suggested [16]. However, Tavallaee [12] didn't find any association between parity and dysmenorrhea.

In our study, the prevalence of women with dysmenorrhea using a contraceptive method was $34.3 \%$. Lower proportions were reported by Sidi [17] and Grandi [18] respectively 9.1\% and 28\%. Tavallaee [12] and Tshabu Aguemon [11] had found higher proportions, respectively $66 \%$ and $47.6 \%$. Few women with dysmenorrhea in our sample used oral contraceptives (6.9\%). This result is consistent with the thesis that oral contraception suppresses dysmenorrhea when it blocks ovulation, but this is not the case with minidosed-pills [19] [20].

More than one in two women had reported asthenia (68.2\%) as an accompanying sign of their dysmenorrhea. This result is close to that of Singh [21] 70.9\%. Also, more than one in two women reported nervousness as an accompanying sign of their dysmenorrhea (54.4\%). Sidi [17] found a similar result (60.1\%). A higher proportion was reported by Diarra [22]: 87.7\%. Moreover, we identified $32.7 \%$ of absenteeism at work related to dysmenorrhea. Our results are similar to those of Avasarala [23] (36\%) but higher than those of Ortiz [24] and Wong [25] respectively $24.8 \%$ and $21.5 \%$. In contrast, Al-Kindi [26] and Diarra [22] reported higher proportions of absenteeism than ours, $45 \%, 48.3 \%$ respectively.

The differences observed between our results and those of other authors in women with dysmenorrhea can be linked to the differences in the populations studied. The proportion of them who used a contraceptive method had severe dysmenorrhea, accompanying signs such as asthenia or irritability, or absence from work.

The factors associated with dysmenorrhea that we found were age, marital status, parity, level of education, and socio-professional occupation. In the literature, it was rather a family history of dysmenorrhea and the onset of menarche before the age of 13 that was the most commonly reported factors [1].

Despite the considerable impact of dysmenorrhea on social life, few women suffering from dysmenorrhea (12.7\%) had been provided medical care for it. This result is similar to those of Wong [25] and Omidvar [27] who reported $12 \%$ and $14.2 \%$ medical consultation for dysmenorrhea respectively. Furthermore, in our sample, a high proportion of women suffering from dysmenorrhea abstained from treatment (30\%). The low proportion of medical consultation may be due to the fact that most girls and their parents, consider dysmenorrhea as a physio- 
logical problem or fatality [1]. Therefore, there is a real need for information on the pathology and its management among adolescent girls and their parents.

The cluster sampling method is suitable for studies exploring large regions or administrative areas as is the case in this study. By making it possible to determine the minimum number of villages/neighborhoods needed to obtain a sufficient sample size suitable for statistical testing for this type of study, it helps to increase accuracy. This type of sampling made it possible to minimize selection bias. The selected sample was representative of the target population of women of childbearing age in the city of Parakou, a city in Northern Benin. Socio-cultural characteristics are not homogeneous throughout Benin territory. The results should be grossed up to women of reproductive age from Benin with some reservations.

\section{Conclusion}

The prevalence of dysmenorrhea is particularly high in the city of Parakou. We have determined some factors associated with this condition. The proportion of women in care is low. It would be important to raise awareness among women for medical and psychosocial care that takes into account the associated factors.

\section{Conflicts of Interest}

The authors declare no conflicts of interest regarding the publication of this paper.

\section{References}

[1] Quereux, C., Graesslin, O. and Gabriel, R. (2004) Les dysménorrhées. In: Blanc, B., Jamin, C. and Sultan, C., Eds., Traité de gynécologie médicale, Springer, Paris, 135-152.

[2] Svanberg, L. and Ulmsten, U. (1981) The Incidence of Primary Dysmenorrhea in Teenagers. Archives of Gynecology, 230, 173-177. https://doi.org/10.1007/BF02111800

[3] Jamieson, D.J. and Steege, J.F. (1996) The Prevalence of Dysmenorrhea, Dyspareunia, Pelvic Pain, and Irritable Bowel Syndrome in Primary Care Practices. Obstetrics \& Gynecology, 87, 55-58. https://doi.org/10.1016/0029-7844(95)00360-6

[4] Elia, D. and Leridon, H. (1996) Epidémiologie de la dysménorrhée: De nouvelles enquêtes en France. Reproduction Humaine et Hormones, 171, 2-4.

[5] Kazama, M., Maruyama, K. and Nakamura, K. (2015) Prevalence of Dysmenorrhea and Its Correlating Lifestyle Factors in Japanese Female Junior High School Students. The Tohoku Journal of Experimental Medicine, 236, 107-113. https://doi.org/10.1620/tjem.236.107

[6] Habibi, N., Huang, M.S.L., Gan, W.Y., Zulida, R. and Safavi, S.M. (2015) Prevalence of Primary Dysmenorrhea and Factors Associated with Its Intensity among Undergraduate Students: A Cross-Sectional Study. Pain Management Nursing, 16, 855-861. https://doi.org/10.1016/j.pmn.2015.07.001

[7] Unsal, A., Ayranci, U., Tozun, M., Arslan, G. and Calik, E. (2010) Prevalence of Dysmenorrhea and Its Effect on Quality of Life among a Group of Female Univer- 
sity Students. Upsala Journal of Medical Sciences, 115, 138-145. https://doi.org/10.3109/03009730903457218

[8] Gumanga, S.K. and Kwame Aryee, R.A. (2012) Menstrual Characteristics in Some Adolescent Girls in Accra, Ghana. Ghana Medical Journal, 46, 3-7.

[9] Jang, I.A., Kim, M.Y., Lee, S.R., Jeong, K.A. and Chung, H.W. (2013) Factors Related to Dysmenorrhea among Vietnamese and Vietnamese Marriage Immigrant Women in South Korea. Obstetrics \& Gynecology Science, 56, 242-248. https://doi.org/10.5468/ogs.2013.56.4.242

[10] Baghianimoghadam, M.H., Loo, A.M., Falahzadeh, H. and Alavijeh, M.M. (2012) A Survey about the Prevalence of Dysmenorrhea in Female Students of Shahid Sadoughi University of Medical Sciences and Their Knowledge, and Practice toward It. Journal of Health Research, 1, 26-31.

[11] Tshabu Aguemon, C., Yunga, F.J.-D., Hounkponou, F., Kpokoun, E., Takpara, I. and Adisso, S. (2016) Dysmenorrhea in School Area in Benin. SOJ Gynecology, Obstetrics \& Women's Health, 2, 1-4. https://doi.org/10.15226/2381-2915/2/1/00107

[12] Tavallaee, M., Joffres, M.R., Corber, S.J., Bayanzadeh, M. and Rad, M.M. (2011) The Prevalence of Menstrual Pain and Associated Risk Factors among Iranian Women. Journal of Obstetrics and Gynaecology Research, 37, 442-451. https://doi.org/10.1111/j.1447-0756.2010.01362.x

[13] László, K.D., Gyorffy, Z., Adám, S., Csoboth, C. and Kopp, M.S. (2008) Work-Related Stress Factors and Menstrual Pain: A Nation-Wide Representative Survey. Journal of Psychosomatic Obstetrics \& Gynecology, 29, 133-138. https://doi.org/10.1080/01674820701804423

[14] Nohara, M. and Momoeda, M.K.T. (2011) Menstrual Cycle and Menstrual Pain Problems and Related Risk Factors among Japanese Female Workers. Industrial Health, 49, 228-234. https://doi.org/10.2486/indhealth.MS1047

[15] Wang, L. and Wang, X.W.W. (2004) Stress and Dysmenorrhea: A Population Based Prospective Study. Occupational and Environmental Medicine, 61, 1021-1026. https://doi.org/10.1136/oem.2003.012302

[16] Juang, C.M., Yen, M.S., Twu, N.F., Horng, H.C., Yu, H.C. and Chen, C.Y. (2006) Impact of Pregnancy on Primary Dysmenorrhea. International Journal of Gynecology \& Obstetrics, 92, 221-227. https://doi.org/10.1016/j.ijgo.2005.12.006

[17] Sidi, I., Hounkpatin, B. and Obossou, A. (2016) Primary Dysmenorrhea in the Schools of Parakou: Prevalence, Impact and Therapeutic Approach. Obstetrics \& Gynecology, 6, 37. https://doi.org/10.4172/2161-0932.1000376

[18] Grandi, G., Ferrari, S., Xholli, A., Cannoletta, M., Palma, F. and Romani, C. (2012) Prevalence of Menstrual Pain in Young Women: What Is Dysmenorrhea? Journal of Pain Research, 5, 169-174. https://doi.org/10.2147/JPR.S30602

[19] Serfaty, D. (2011) Contraception. 4th Edition, Elsevier-Masson, Issy-les-Moulineaux, $592 \mathrm{p}$.

[20] Andersh, B. and Milsom, I. (1982) An Epidemiologic Study of Young Women with Dysmenorrhea. American Journal of Obstetrics \& Gynecology, 144, 655-660. https://doi.org/10.1016/0002-9378(82)90433-1

[21] Singh, A., Kiran, D., Singh, H., Nel, B., Singh, P. and Tiwari, P. (2008) Prevalence and Severity of Dysmenorrhea: A Problem Related to Menstruation, among First and Second Year Female Medical Students. Indian Journal of Physiology and Pharmacology, 52, 389-397.

[22] Diarra, I., Kouyaté, Y.M., Sow, S.A. and Maiga, M.K. (2009) Dysmenorrhea in Ado- 
lescents: Epidemio-Clinical Study at the Referral Health Center, Bamako District. Report of 300 Cases. Le Mali Médical, 24, 55-58.

[23] Avasarala, A.K. and Panchangam, S. (2008) Dysmenorrhea in Different Settings: Are the Rural and Urban Adolescent Girls Perceiving and Managing the Dysmenorrhea Problem Differently? Indian Journal of Community Medicine, 33, 246-249. https://doi.org/10.4103/0970-0218.43231

[24] Ortiz, M.I., Rangel-Flores, E., Carrillo-Alarcón, L.C. and Veras-Godoy, H.A. (2009) Prevalence and Impact of Primary Dysmenorrhea among Mexican High School Students. International Journal of Gynecology \& Obstetrics, 107, 240-243. https://doi.org/10.1016/j.ijgo.2009.07.031

[25] Wong, L.P. and Khoo, E.M. (2009) Dysmenorrhea in a Multiethnic Population of Adolescent Asian Girls. International Journal of Gynecology \& Obstetrics, 108, 139-142. https://doi.org/10.1016/j.ijgo.2009.09.018

[26] Al-Kindi, R. and Al-Bulushi, A. (2011) Prevalence and Impact of Dysmenorrhea among Omani High School Students. Sultan Qaboos University Medical Journal, 11, 485-491.

[27] Omidvar, S., Bakouei, F., Amiri, F.N. and Begum, K. (2016) Primary Dysmenorrhea and Menstrual Symptoms in Indian Female Students: Prevalence, Impact and Management. Global Journal of Health Science, 8, 135-144.

https://doi.org/10.5539/gjhs.v8n8p135 